\title{
Medición de Campos Electromagnéticos en la Ciudad de Cali, Colombia
}

\author{
Guillermo Aponte $^{(1)}$, Adolfo Escobar ${ }^{(1)}$, Carlos R. Pinedo $^{(1)}$ y Gisela Arizabaleta ${ }^{(2)}$ \\ (1) Universidad del Valle. Escuela de Ingeniería Eléctrica y Electrónica, Ciudad Universitaria \\ Meléndez, Calle 13 No. 100-00. Cali-Colombia (e-mail: gponte@univalle.edu.co) \\ (2) Departamento Administrativo de Gestión del Medio Ambiente DAGMA, Av. 5AN No. 20N-08, \\ Edif. Fuente de Versalles Piso 10. Cali-Colombia (e-mail: garizabaleta@dagma.com.co)
}

\begin{abstract}
Resumen
En este artículo se presenta una metodología de medición del campo electromagnético producido por instalaciones de radio y telecomunicaciones y se presentan los resultados obtenidos en la evaluación de 50 sitios en la ciudad de Cali-Colombia. Este trabajo se originó por la preocupación pública causada por la proliferación de estaciones base de telefonía celular en el ámbito urbano y por los temores acerca de los posibles efectos nocivos de los campos electromagnéticos en la salud. La evaluación de los sitios se realizó con el propósito de conocer y cuantificar los niveles de campo electromagnético existentes en el medio ambiente de la ciudad. En general, los valores encontrados fueron inferiores al $0.5 \%$ del límite establecido por la Comisión Internacional para la Protección de las Radiaciones No lonizantes, ICNIRP $\left(0.2 \mathrm{~mW} / \mathrm{cm}^{2}\right)$. Sin embargo, en dos casos los niveles encontrados superaron dicho límite.
\end{abstract}

Palabras clave: campo electromagnético, estaciones base, telefonía celular, medición

\section{Electromagnetic Field Measurement in the City of Cali, Colombia}

\begin{abstract}
This paper shows a measurement methodology of the electromagnetic field produced by radio and telecommunications installations and presents results obtained in the evaluation of 50 measurement sites in the city of Cali in Colombia. This work was done because people's concern about proliferation of radio base stations in urban areas, and their fear about the possible dangerous effects of the electromagnetic fields on human health. The evaluation was carried out to determine and quantify the levels of electromagnetic field that exist in the environment of the city. In general, the values found were lower than $0.5 \%$ of the limit established by the International Commission for Non-Ionizing Radiation Protection, ICNIRP $\left(0.2 \mathrm{~mW} / \mathrm{cm}^{2}\right)$. However, in two sites the measured values exceeded this limit.
\end{abstract}

Keywords: electromagnetic field, base station, mobile phone, measurement. 


\section{INTRODUCCIÓN}

Para satisfacer las necesidades de sus usuarios, los operadores de los sistemas de comunicaciones utilizan las ondas electromagnéticas, e instalan una tupida red de estaciones base, logrando así una buena cobertura, que posibilita comunicarse prácticamente desde cualquier lugar. La proliferación de estas estaciones base en el ámbito urbano ha despertado gran preocupación en la sociedad por los posibles riesgos adversos para la salud debidos a los campos electromagnéticos de alta frecuencia generados por los sistemas de AM, FM, microondas, telefonía fija inalámbrica y telefonía celular (Cruz, 2005); siendo este es un tema de actualidad sobre el cual se ha creado mucha especulación.

Sobre este tema se encendió una luz de alerta a la que acuden presurosos tanto los organismos gubernamentales como los propietarias de las instalaciones, las organizaciones de vecinos, ecologistas, etc., con un gran interés de hacer claridad sobre los riesgos, niveles permitidos, procedimientos de evaluación (Bruni, et al., 2003; Giuliani, 2003) y verificación de las emisiones electromagnéticas (Galvao et al., 2001). El organismo encargado del Medio Ambiente de la Ciudad de Cali (DAGMA) y la Universidad del Valle, desarrollaron un proyecto para conocer los valores máximos de los campos electromagnéticos de alta frecuencia a los cuales están diariamente expuestos las personas en la ciudad y verificar si estos valores pueden o no considerarse como aceptables.

En este proyecto, se efectuaron mediciones en 50 puntos distribuidos en distintas zonas de la ciudad, con el propósito de conocer y cuantificar los niveles de inmisión de campo electromagnético existentes. El trabajo se desarrolló con rigurosidad metodológica, implementando procedimientos de medición de acuerdo a recomendaciones y normas internacionales (CNC, 2003; IEEE, 2002).

\section{LOS CAMPOS ELECTROMAGNÉTICOS}

Las ondas electromagnéticas se componen simultáneamente como su nombre lo indica, de un campo eléctrico y un campo magnético (Fig. 1).

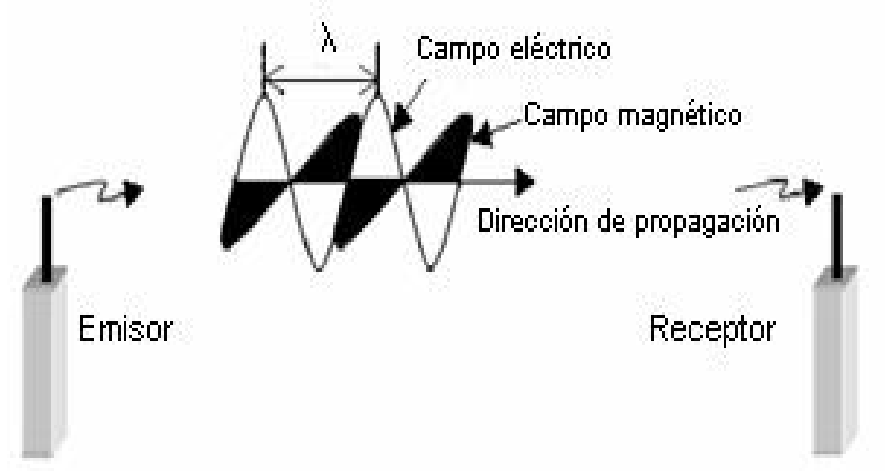

Fig. 1: Propagación de ondas en comunicaciones

La intensidad de los campos alrededor de una fuente de radiación depende estrechamente de la potencia radiada y de la distancia a la fuente. De acuerdo con las propiedades dieléctricas de los objetos, la energía de la onda es reflejada, refractada, difractada, dispersada y absorbida por tales objetos (Portela et al., 1998). Por su velocidad de cambio o frecuencia, las ondas pueden clasificarse dentro de un espectro electromagnético que va desde frecuencias extremadamente bajas, donde están los sistemas eléctricos, hasta las muy altas correspondientes a la radiación cósmica y los rayos gama (Fig. 2). Por los fenómenos físicos que pueden ocasionar las radiaciones, el espectro se encuentra dividido en dos regiones: la de radiaciones no-ionizantes y la de radiaciones ionizantes (para frecuencias a partir de $10^{15} \mathrm{~Hz}$.) En la primera región los efectos de la energía asociada a la radiación son demasiado débiles para romper los enlaces que mantienen unidas las moléculas de las células, mientras que en la segunda se produce ionización en la materia, es decir, la energía asociada puede romper los enlaces de las moléculas. 


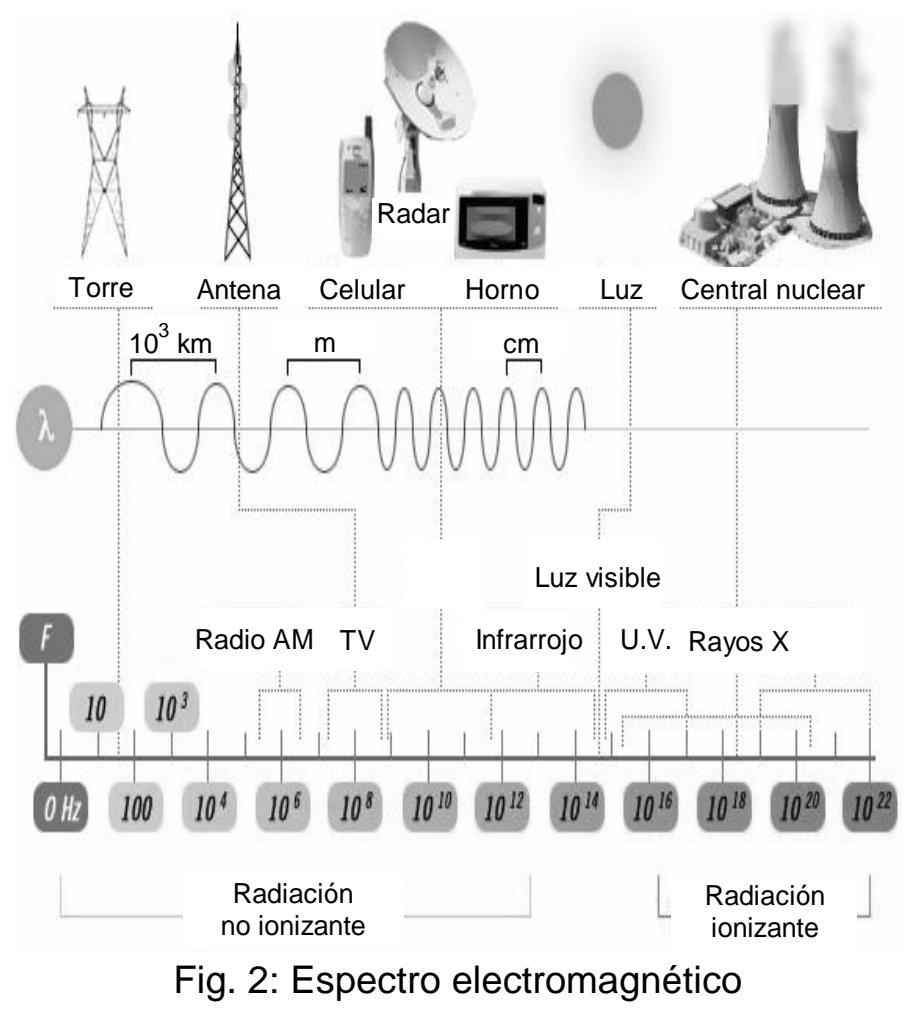

Una parte del espectro es denominada radiofrecuencia y está conformada por ondas con frecuencias entre $3 \mathrm{kHz}$ y $300 \mathrm{GHz}$, estando allí los sistemas de comunicación de radio AM y FM, televisión, telefonía inalámbrica, telefonía celular, comunicación personal, radioaficionados, radio enlaces y microondas (González, 1999).

\section{POSIBLES EFECTOS DE LOS CAMPOS}

La vida en la tierra está sustentada por parámetros ambientales muy bien definidos como son: concentración de oxígeno, presión atmosférica, temperatura, vapor de agua, luz. Estos deben mantenerse dentro de ciertos "límites normales", dado que ante pequeñas desviaciones el organismo responde fisiológicamente.

Existen otros factores no detectables por el organismo, como el campo magnético terrestre, que pueden también influir en los organismos, esto se evidencia en la existencia de algunos "biociclos" asociados con las variaciones del campo. Los campos naturales son perfectamente tolerables, dado que la evolución de todos los seres, incluido el hombre, se produjo en su presencia. Existen también campos artificiales o generados por el hombre, que suelen tener intensidades superiores a las de los naturales y ante los cuales ya no es dable esperar la misma adaptabilidad de los organismos, agravado por el hecho de que los animales de orden superior no tienen modo de detectarlos.

Por falta de evidencias no puede deducirse que los seres no son afectados, por el contrario podría afirmarse que existen efectos, lo importante es determinar si los mismos pueden ser beneficiosos, nocivos o inocuos. Entre los factores que influyen para que una persona pueda soportar cierta cantidad de radiación electromagnética sin efectos perjudiciales, están la temperatura y humedad del ambiente, la cantidad de potencia ya absorbida en exposiciones anteriores, la sensibilidad y el estado de salud general (Álvarez et al., 2000). En cada caso debe tenerse en cuenta que lo importante es el nivel de campo que llega y el grado de absorción del mismo, lo cual es difícil de prever dado que las configuraciones de los seres vivos son sumamente complejas y diferentes.

El efecto de la radiación en el interior de un tejido vivo, depende de la frecuencia de la onda, su intensidad y polarización; del tamaño, forma y propiedades eléctricas del elemento expuesto; de la presencia o ausencia de otros elementos en la vecindad capaces de reflejar señal y finalmente, de la posición relativa o contacto con el plano de tierra (Álvarez et al., 2001). 
La relación entre los campos electromagnéticos y la aparición de efectos directos como el cáncer, ha sido y sigue siendo, materia de muchos estudios serios. Esos estudios, en muchos casos, contienen posibles "factores de confusión", ya que no garantizan el total control de otras variables como estado socioeconómico, tipo de vivienda y alimentación, diferencia entre zonas urbanas y rurales, movilidad familiar, presencia de agentes químicos y factores genéticos, que pueden tener influencia en las conclusiones (MSC, 1999).

\section{METODOLOGÍA DE MEDICIÓN}

Para la medición del campo electromagnético se deben tener en cuenta algunos factores importantes como: características del sitio, distancia a la fuente de radiación, las variables a medir, las unidades de medida, el tipo de medición; además, se debe estar familiarizado con las normas relacionadas con este tipo de mediciones. Igualmente se deben conocer los límites de exposición permitidos, contar con los instrumentos de medición requeridos y conocer el patrón de radiación de la fuente (Fig. 3).

Un campo electromagnético cuenta con dos componentes, una eléctrica y otra magnética, el campo eléctrico se mide habitualmente en voltios por metro $(\mathrm{V} / \mathrm{m})$ y el magnético en amperios por metro $(\mathrm{A} / \mathrm{m}$.) La densidad de potencia, que es la combinación de los dos componentes, se mide en miliwatios por centímetro cuadrado ( $\mathrm{mW} / \mathrm{cm} 2)$, siendo esta última mucho más útil cuando el lugar de medida está muy lejos de la fuente emisora, zona que se conoce como de campo lejano. En campo lejano, el campo eléctrico $\mathrm{E}$ y el magnético $\mathrm{H}$, se encuentran relacionados de manera conocida $(\mathrm{E}=$ $\mathrm{Z} \times \mathrm{H}$ ), por lo que conociendo una de estas magnitudes, queda definida la otra y, por tanto, la densidad de potencia. En la zona de campo cercano (zona cercana a la fuente) se hace necesaria la medición de ambas componentes de campo para poder caracterizarlo completamente.

\section{Medición de emisión}

Medición del valor promedio de la intensidad de campo eléctrico o magnético debidos a una fuente de radiofrecuencia determinada, la cual opera a una frecuencia específica. Para ello se utilizan equipos de medición en banda angosta.

\section{Medición de inmisión}

Medición del campo electromagnético producto del aporte de múltiples fuentes de radiofrecuencia, que operan a distintas frecuencias. En este tipo de mediciones se utilizan equipos de banda ancha. Para evaluar la exposición poblacional se realiza la medición de inmisión, ya que las personas están expuestas simultáneamente a muchas fuentes de radiación. En el proyecto se empleó un medidor de inmisión de campo electromagnético isotrópico, modelo EMR-300 de la marca NARDA (Fig. 4). Este medidor utiliza diferentes sondas de medición, dependiendo de si se desea medir el campo eléctrico o el magnético y de la banda de frecuencia en la que se desea realizar la medición. Para hacer la medición, previamente se debe recorrer el sitio con el objetivo de reconocer el tipo de instalación y determinar los puntos de mayor interés para medir. Los aspectos a tener en cuenta durante este reconocimiento son los siguientes:

Primero, identificar las zonas accesibles para el público en general, próximas a la antena radiante más cercana. Luego, verificar si existen lugares de residencia habitual, a distancias cortas de las antenas radiantes, especialmente en la dirección de máxima radiación. Después de esto, observar si se encuentran edificios u otros obstáculos, estimando de qué manera su presencia puede afectar al proceso de medida, fundamentalmente debido a reflexiones; comprobar si existen escuelas, hospitales, parques públicos, etc., situados en lugares próximos a las estaciones base. Por último, identificar las fuentes de señal radioeléctrica en las inmediaciones del entorno de medida y su posible incidencia en la medición.

Las mediciones deben considerar las cuatro direcciones ortogonales a partir de la estación base (perfiles), como se observa en la Fig. 5 y distancias de aproximadamente 2, 12, 50 y 100 metros desde el soporte de la estación base. El medidor debe estar a una altura de 1.8 metros sobre el suelo y se debe dejar midiendo por un período de 6 minutos. 


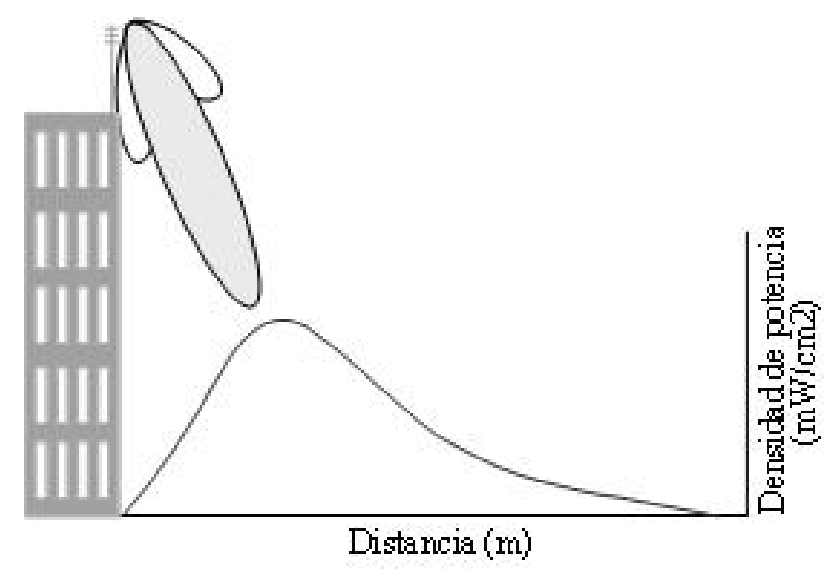

Fig. 3: Perfil típico del campo emitido por una estación base de telefonía celular

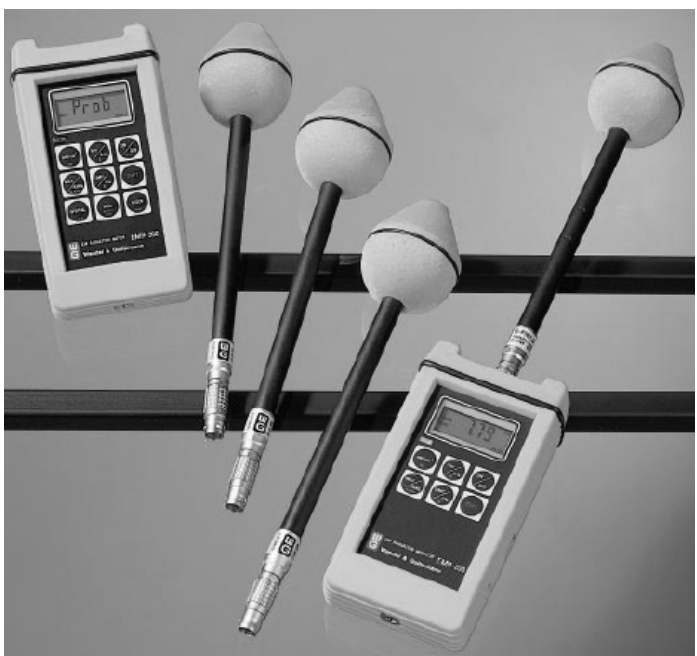

Fig. 4: Medidor de banda ancha

Los anteriores requerimientos se pueden cumplir cuando se mide un sistema que radia de manera omnidireccional y situado en campo despejado, como es el caso de las emisoras de radio AM, generalmente ubicadas en las afueras de la ciudad. Cuando se realizan mediciones dentro de la ciudad, por ejemplo sobre estaciones de telefonía celular y de radio FM, las distancias y la dirección de los puntos de medición están condicionadas por la ubicación del sistema (direcciones en que está radiando) y por los lugares expuestos a radiación. Se puede también realizar mediciones a alturas diferentes a 1.8 metros, tal es el caso de las escuelas, donde 1 metro puede ser un valor acorde con la altura promedio de los niños.

Durante el proyecto se efectuaron mediciones en 50 sitios distribuidos en distintas zonas de la ciudad de Cali (Fig. 6), con el propósito de conocer y cuantificar los niveles de inmisión de campo electromagnético en estos puntos, para tener una muestra representativa de los niveles de campo electromagnético existentes en la ciudad. Los 50 sitios se escogieron, de un listado de 200 lugares, entregado por la Personería Municipal de la ciudad, donde a finales del año 2004 se encontraban estaciones base de telecomunicaciones.

En la Tabla 1 se describen los sitios en donde se realizaron las mediciones. Algunos de ellos eran de interés especial, ya que se encontraban dentro o cerca de escuelas, hospitales o lugares representativos de la ciudad. La Tabla 2 muestra una distribución del número de sistemas medidos de acuerdo a su tipo y cantidad.

\section{REGULACIONES}

De acuerdo con la literatura científica, no hay hasta el momento estudios que permitan tener completa certeza sobre si los campos electromagnéticos tienen o no efectos sobre la salud humana, sin embargo, existen recomendaciones internacionales acerca de los límites de exposición a estos campos para las personas (IEEE, 1999; UIT, 1998).

\section{Recomendación del ICNIRP}

Las pautas dadas por la Comisión Internacional para la Protección de las Radiaciones No lonizantes (ICNIRP, 1998), una organización científica independiente y reconocida en el ámbito mundial, para limitar la exposición a campos electromagnéticos, contemplan dos tipos de exposición: la ocupacional y la del público en general. 


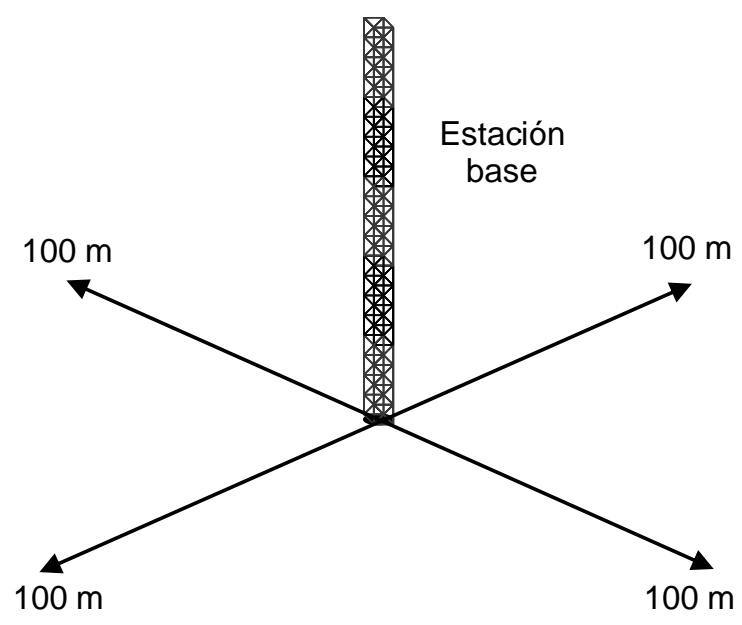

Fig. 5: Perfiles de medición

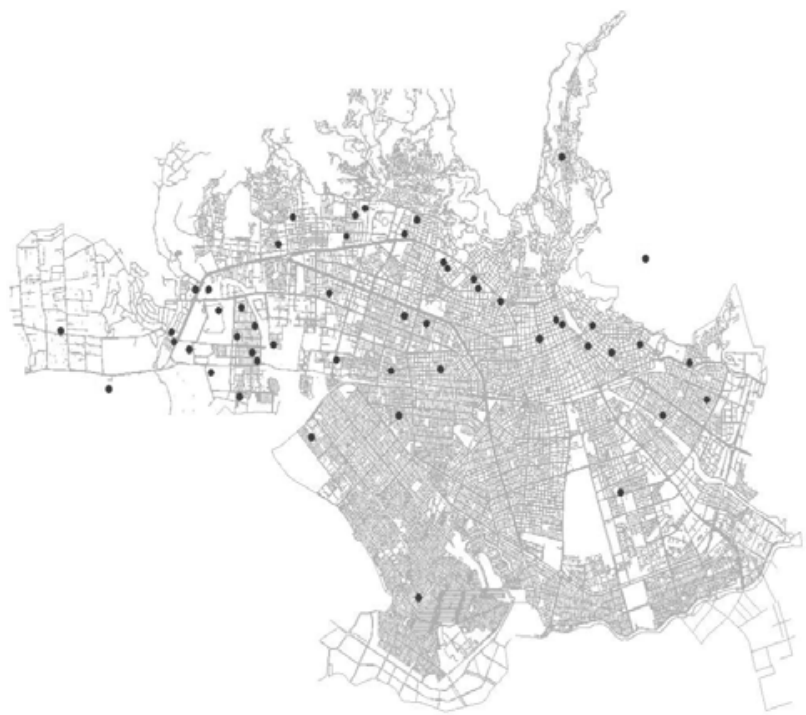

Fig. 6: Puntos de medición

Tabla 1. Descripción de los sitios de medición

\begin{tabular}{|l|c|c|}
\hline \multicolumn{1}{|c|}{ Lugar } & Cantidad & Porcentaje \\
\hline Campo despejado & 2 & $4 \%$ \\
\hline Azotea & 7 & $14 \%$ \\
\hline De interés especial & 12 & $24 \%$ \\
\hline Calle & 29 & $58 \%$ \\
\hline
\end{tabular}

Tabla 2. Descripción de los sistemas medidos

\begin{tabular}{|l|c|c|}
\hline \multicolumn{1}{|c|}{ Sistema medido } & Cantidad & Porcentaje \\
\hline Radio AM & 1 & $2 \%$ \\
\hline Múltiples & 1 & $2 \%$ \\
\hline Radio FM & 2 & $4 \%$ \\
\hline Radio taxis & 2 & $4 \%$ \\
\hline Fija inalámbrica (FI) & 9 & $18 \%$ \\
\hline Celular y FI & 10 & $20 \%$ \\
\hline Celular & 25 & $50 \%$ \\
\hline
\end{tabular}

La exposición ocupacional consiste en la que reciben, bajo condiciones conocidas y por periodos cortos, adultos que han sido entrenados al respecto, que conocen bien el riesgo potencial y saben tomar las precauciones apropiadas. En contraste, la exposición poblacional es la ocasionada sobre grupos de individuos de todas las edades y estados de salud, que no tienen conocimiento de su exposición, la cual ocurre de manera continua.

Normativa Colombiana

El decreto colombiano (MINCOM, 2005), definió los límites (Tabla 3) de exposición de las personas a campos electromagnéticos en Colombia.

Los límites adoptados en este decreto, se basaron en las recomendaciones del ICNIRP. En la Fig. 7 se presentan los valores límites de densidad de potencia, establecidos en el decreto reglamentario. 


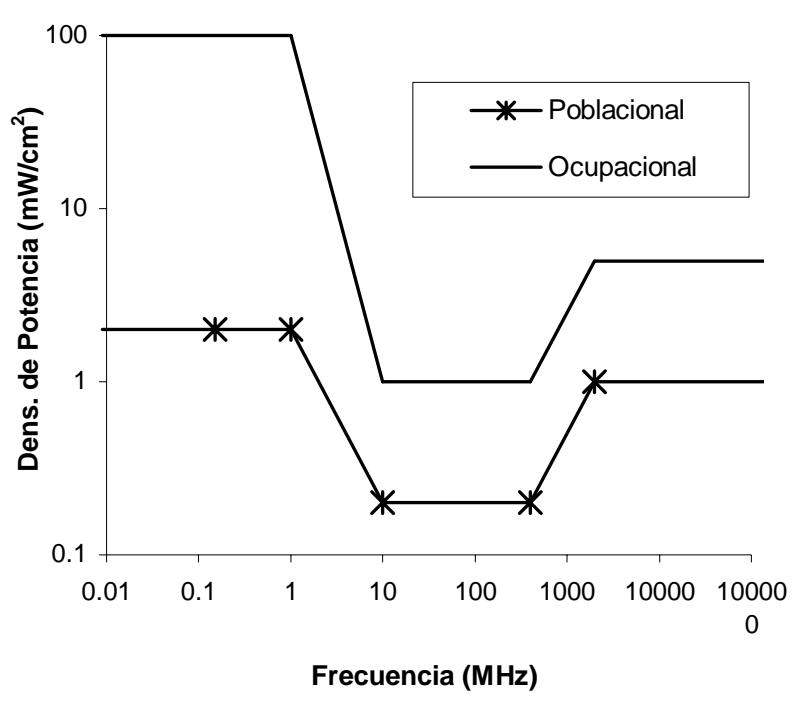

Fig. 7: Límites para densidad de potencia

\section{RESULTADOS DE LAS MEDICIONES}

En la Fig. 8 se presenta un ejemplo de la gráfica de variación de la densidad de potencia $\left(\mathrm{mW} / \mathrm{cm}^{2}\right)$ en función del tiempo, obtenida para un punto en particular y la Fig. 9 muestra los valores máximos y promedios en todos los puntos, de uno de los 50 sitios de medición. Los valores registrados de las mediciones efectuadas, se compararon contra el nivel más bajo de densidad de potencia $(0.2$ $\mathrm{mW} / \mathrm{cm}^{2}$ ), indicado en el Decreto Colombiano. La Fig. 10 muestra los valores obtenidos en los 50 sitios y su porcentaje con respecto al valor más bajo.

En 24 de los 50 sitios, se encontró que los valores medidos estaban entre el 0.1 y el $0.5 \%$ del nivel de referencia de $0.2 \mathrm{~mW} / \mathrm{cm}^{2}$. Por otro lado, se encontró que en dos de los 50 sitios medidos, los niveles de campo electromagnético superaron dicho nivel. Un sitio correspondía a un sistema de telefonía celular y el otro a un sistema de radio AM. La Fig. 11 muestra los valores encontrados en 25 estaciones base de telefonía celular medidas y su relación con el valor de referencia. Solamente se encontró un sitio donde los valores fueron superiores al límite de $0,2 \mathrm{~mW} / \mathrm{cm}^{2}$.

Tabla 3: Límites de exposición en Colombia

\begin{tabular}{|c|c|c|c|c|}
\hline $\begin{array}{c}\text { Tipo de } \\
\text { exposición }\end{array}$ & $\begin{array}{c}\text { Frecuencia } \\
(\mathrm{MHz})\end{array}$ & $\begin{array}{c}\text { Campo eléctrico } \\
\mathrm{rms}(\mathrm{V} / \mathrm{m})\end{array}$ & $\begin{array}{c}\text { Campo magnético } \\
\mathrm{rms}(\mathrm{A} / \mathrm{m})\end{array}$ & $\begin{array}{c}\text { Densidad de } \\
\text { potencia }\left(\mathrm{W} / \mathrm{m}^{2}\right)\end{array}$ \\
\hline \multirow{5}{*}{ Ocupacional } & $0.009-0.065$ & 610 & 24,4 & - \\
\cline { 2 - 5 } & $0.065-1$ & 610 & $1,6 / \mathrm{f}$ & - \\
\cline { 2 - 5 } & $1-10$ & $610 / \mathrm{f}$ & $1,6 / \mathrm{f}$ & - \\
\cline { 2 - 5 } & $10-400$ & 61 & 0,16 & $\mathrm{f} / 40$ \\
\cline { 2 - 5 } & $400-2000$ & $3 \mathrm{f}^{1 / 2}$ & $0,008 \mathrm{f}^{1 / 2}$ & 50 \\
\cline { 2 - 5 } & $2000-300000$ & 137 & 0,36 & - \\
\hline \multirow{5}{*}{$\begin{array}{c}\text { Público en } \\
\text { general }\end{array}$} & $0.009-0.15$ & 87 & 5 & - \\
\cline { 2 - 5 } & $0.15-1$ & 87 & $0,73 / \mathrm{f}^{1 / 2}$ & 2 \\
\cline { 2 - 5 } & $1-10$ & $87 / \mathrm{f}^{1 / 2}$ & 0,073 & $\mathrm{f} / 200$ \\
\cline { 2 - 5 } & $400-2000$ & $1,375 \mathrm{f}^{1 / 2}$ & $0,0037 \mathrm{f}^{1 / 2}$ & 10 \\
\cline { 2 - 5 } & $2000-300000$ & 61 & 0,16 & - \\
\hline
\end{tabular}




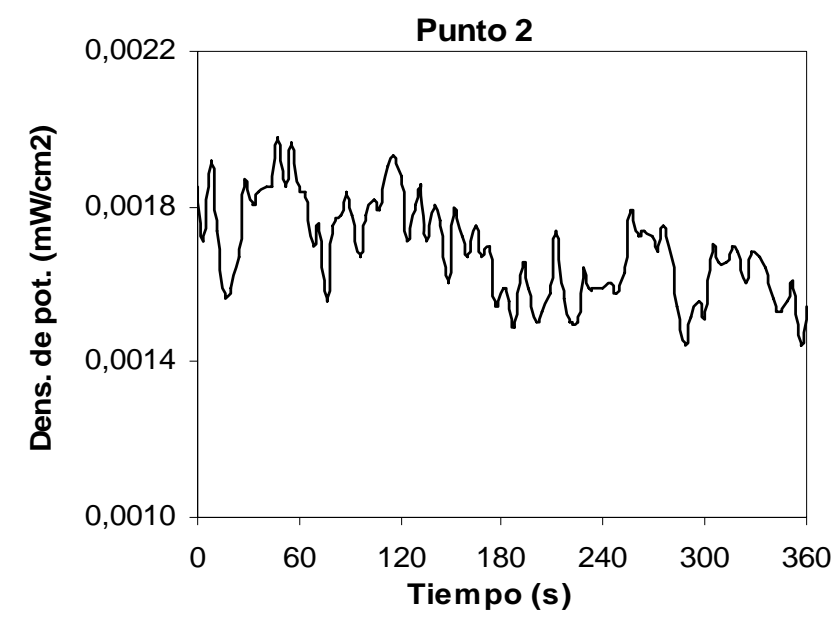

Fig. 8: Densidad de potencia medida en función del tiempo para un punto

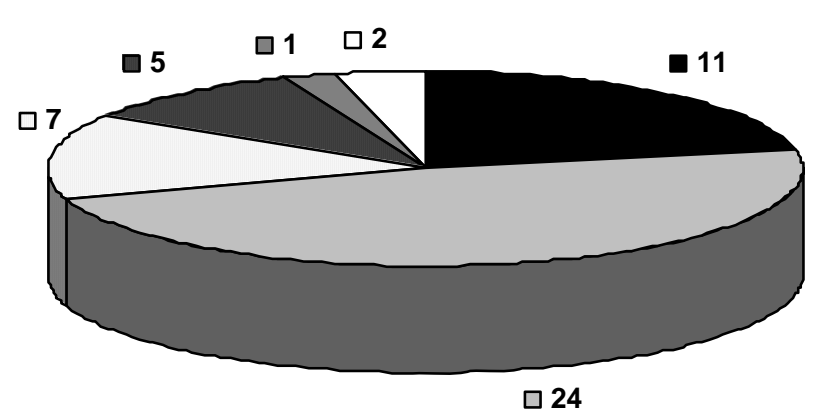

- Menores al $0.1 \%(11) \quad \square$ Entre 0.1 y $0.5 \%(24)$

口Entre 0.5 y $1 \%(7)$

$\square$ Entre 1 y $5 \%$ (5)

口Entre 5 y $100 \%$ (1)

口Mayores al 100\% (2)

Fig. 10: Valores máximos medidos con respecto a $0.2 \mathrm{~mW} / \mathrm{cm}^{2}$

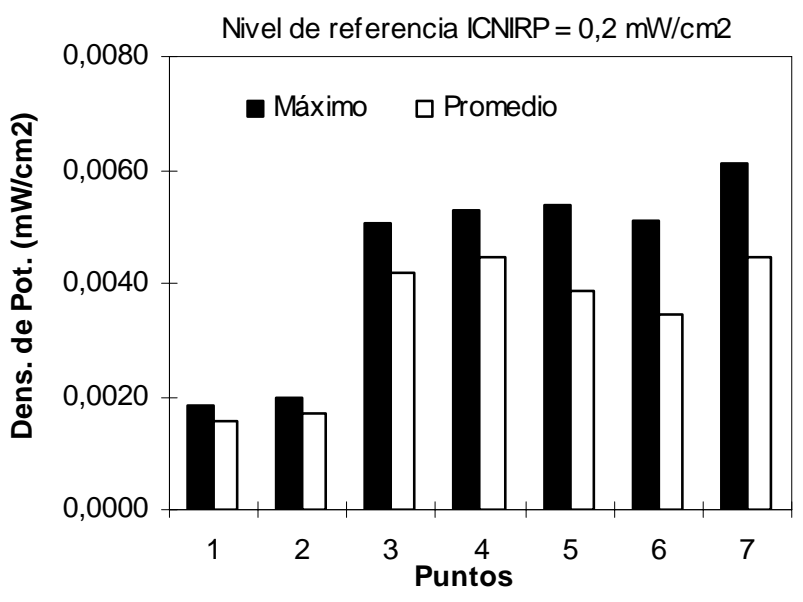

Fig. 9: Valores máximos y promedios medidos para todos los puntos en un sitio

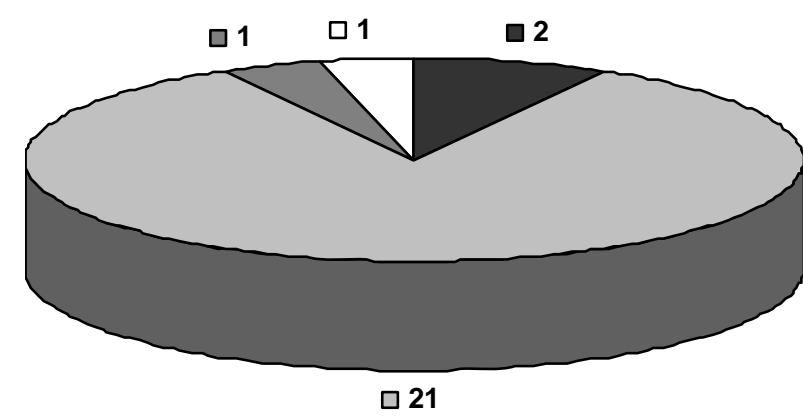

$\square$ Menores al $0.5 \%(2) \quad \square$ Entre 0.5 y $1 \%(21)$

$\square$ Entre 1 y $5 \%(1) \quad \square$ Mayores al $100 \%$ (1)

Fig. 11: Valores máximos en sistemas de telefonía celular con respecto a $0.2 \mathrm{~mW} / \mathrm{cm}^{2}$

\section{CONCLUSIONES}

La evaluación de los niveles de inmisión de densidad de potencia en 50 sitios en la Ciudad de Cali, muestra que el panorama general es bueno, ya que la gran mayoría (96\%), los valores están muy por debajo de los límites permitidos. Se encontró que en dos de los 50 sitios medidos, los niveles de campo electromagnético superaron los niveles establecidos en el Decreto Colombiano. En estos sitios se podría prevenir la exposición de las personas a niveles altos de campo electromagnético, simplemente colocando un encerramiento alrededor de las estaciones base, con el fin de limitar el acceso.

\section{AGRADECIMIENTOS}

Los autores agradecen al DAGMA por su aporte económico y al ingeniero Claudio Muñoz del Centro Argentino de Estudios de Radiocomuni-caciones y Compatibilidad Electromagnética CAERCEM, por su asesoría en el proyecto. 


\section{REFERENCIAS}

Álvarez, B.; Padula, V.; Saint R. y Muñoz, C., Reflexiones acerca de la Incidencia de los Campos Electromagnéticos en la Salud Humana, (en línea), www.itba.edu.ar/caercem, Argentina (2000).

Álvarez, B.; Padula, V.; Saint R. y Muñoz, C., Radiaciones no Ionizantes: Efectos Biológicos en las Bandas de Telefonía Celular, (en línea), www.itba.edu.ar/caercem, Argentina (2001).

Bruni, R.; Dujovne, D.; Vanilla, O. y Taborda, R. Evaluación de Radiación Electromagnética de Fuentes no Naturales, XIV Congreso Argentino de Bioingeniería y III Jornadas de Ingeniería Clínica SABI'2003, Córdoba, Argentina (2003).

CNC, Comisión Nacional de Comunicaciones. Resolución 117/2003: Evaluación Previa a la Instalación de Antenas Emisoras de Conformidad con los Parámetros de la Res. 202/95, Argentina (2003).

Cruz, V., Mediciones y Evaluación de las Radiaciones no Ionizantes de Cuarenta Estaciones Bases de Servicios Móviles en la Ciudad de Lima, Revista AHCIET Móvil, No. 3, Septiembre (2005).

Galvao, B.S.M.C.; Santos, G.; Onusic, H. y Sant'Anna, L., Electromagnetic Environmental Measurements in Specific Populated Areas of Brazil, IEEE International Symposium on Electromagnetic Compatibility, EMC'2001, Montreal, Canadá (2001).

Giuliani, L. et al. Environmental investigation on the electromagnetic field levels in the urban area of Rome, 3rd International EMF Seminar in China, Guilin, China (2003).

González, A.; Morcillo, A. y Hernández, D., Exposición Humana a Campos Electromagnéticos, Universidad Politécnica de Cartagena, Cartagena, España (1999).

IEEE, Std. C95.1, IEEE Standard for Safety Levels with Respect to Human Exposure to Radio Frequency Electromagnetic Fields - $3 \mathrm{kHz}$ to $300 \mathrm{GHz}$, The Institute of Electrical and Electronic Engineers (1999).

IEEE, Std. C95.3, IEEE Recommended Practice for Measurements and Computations of Radio Frequency Electromagnetic Fields With Respect to Human Exposure to Such Fields, $100 \mathrm{kHz}-300 \mathrm{GHz}$, The Institute of Electrical and Electronic Engineers (2002).

ICNIRP, International Commission on Non-Ionizing Radiation Protection, Guidelines for Limiting Exposure to Time-Varying Electric, Magnetic and Electromagnetic Fields (up to $300 \mathrm{GHz}$ ), Health Physics, Vol. 74, No. 4 (1998).

MINCOM, Ministerio de Comunicaciones, Decreto 195 por el cual se adoptan límites de exposición a las personas a campos electromagnéticos, se adecuan procedimientos para la instalación de estaciones radioeléctricas y se dictan otras disposiciones, Norma Colombiana (2005).

MSC, Ministerio de Sanidad y Consumo de España, Campos Electromagnéticos y Salud Pública, Informe técnico elaborado por el comité de expertos, Subdirección general de sanidad ambiental y salud laboral, Dirección general de salud pública y consumo, España (1999).

Portela, A.; Skvarca, J.; Matute, E. y Loureiro, L., Prospección de Radiación Electromagnética Ambiental no Ionizante, Vol. I, Manual de Estándares de Seguridad para la Exposición a Radiofrecuencias Comprendidas entre $100 \mathrm{kHz}$ y $300 \mathrm{GHz}$, Dirección nacional de calidad ambiental, Argentina (1988).

UIT, Unión Internacional de Telecomunicaciones, UIT-T K.52, Orientación sobre el Cumplimiento de los Límites de Exposición de las Personas a los Campos Electromagnéticos, Unión Internacional de Telecomunicaciones (2000). 
